\title{
RS Wave Amplitude Single Beat
}

National Cancer Institute

\section{Source}

National Cancer Institute. RS Wave Amplitude Single Beat. NCI Thesaurus. Code

C117794.

An electrocardiographic measurement of the sum of the amplitudes of the R and S

waves, obtained from a single beat in one particular lead or set of leads. (CDISC) 